\title{
Chloroquine exerts neuroprotection following traumatic brain injury via suppression of inflammation and neuronal autophagic death
}

\author{
CHANG-MENG CUI $^{1}$, JUN-LING GAO $^{2}$, YING CUI $^{3}$, LI-QIAN SUN $^{3}$, YONG-CHAO WANG $^{2}$, \\ KAI-JIE WANG ${ }^{3}$, RAN LI $^{2}$, YAN-XIA TIAN ${ }^{2}$ and JIAN-ZHONG CUI ${ }^{1,3}$ \\ ${ }^{1}$ Department of Surgery, Hebei Medical University, Shijiazhung, Hebei 050017; ${ }^{2}$ School of Basic Medical Science, \\ Hebei United University; ${ }^{3}$ Department of Neurosurgery, Tangshan Workers' Hospital, Tangshan, Hebei 063000, P.R. China
}

Received July 30, 2013; Accepted March 31, 2014

DOI: $10.3892 / \mathrm{mmr} .2015 .3611$

\begin{abstract}
The antimalarial drug, chloroquine (CQ), has been reported as an autophagy inhibitor in a variety of disorders, including Alzheimer's disease and brain ischemia. To the best of our knowledge, no studies to date have examined the potential for CQ to provide neuroprotection in animal models of traumatic brain injury (TBI). The aim of this study was to investigate the neuroprotective actions of CQ in TBI and to determine the mechanisms underlying this effect. Rats were immediately subjected to a diffuse cortical impact injury caused by a modified weight-drop device and divided randomly into three groups: sham-operated, CQ treatment and vehicle. The CQ treatment group was administered CQ (intraperitoneally, $3 \mathrm{mg} / \mathrm{kg}$ body weight) immediately following the induction of injury. The co-localization of neuron-specific nuclear protein $(\mathrm{NeuN})$ and microtubule-associated protein 1 light chain 3 (LC3), was followed by immunofluorescent staining. The expression of LC3 and inflammatory cytokines was identified by western blot analysis. Wet-dry weight method was utilized to evaluate TBI-induced brain edema. Motor function was evaluated using the Neurological Severity Score (NSS) scale and the Morris water maze was employed to assess spatial learning ability. This study demonstrated that the administration of CQ attenuates TBI-induced cerebral edema,
\end{abstract}

Correspondence to: Dr Jian-Zhong Cui, Department of Surgery, Hebei Medical University, No. 361 East Zhongshan Road, Shijiazhuang, Hebei 050017, P.R. China

E-mail: jzhcuidr2001@163.com

Abbreviations: CQ, chloroquine; TBI, traumatic brain injury; LC3, microtubule-associated protein 1 light chain 3 ; NeuN, neuron-specific nuclear protein; DAPI, 4',6-diamidino-2-phenylindole; NSS, Neurologic Severity Score; TNF- $\alpha$, tumor necrosis factor- $\alpha$; IL-1, interleukin-1.

Key words: chloroquine, autophagy, neuroprotection, traumatic brain injury, rats and the associated motor and cognitive functional deficits that occur post-injury. Following the induction of cerebral trauma, CQ treatment significantly suppressed neuronal autophagy and reduced expression levels of the inflammatory cytokines, interleukin-1 $\beta$ (IL-1 $\beta$ ) and tumor necrosis factor- $\alpha$ (TNF- $\alpha$ ), in the rat hippocampus. Our results have provided in vivo evidence that CQ may exert neuroprotective effects following TBI, in attenuating brain edema and improving neurological functioning, by reducing the damaging consequences of neuronal autophagy and cerebral inflammation.

\section{Introduction}

Traumatic brain injury (TBI) is one of the leading causes of morbidity and mortality in young adults and children, and is a leading public health problem worldwide (1). In TBI, neurological impairment is caused by immediate brain tissue disruption (primary injury) and post-injury cellular and molecular events (secondary injury) that exacerbate the primary neurological insult (2). These combined events lead to the induction of mitochondrial dysfunction and the amplification of biochemical cell-death signaling cascades, which cause neuronal cell death and general neurological functional deficits $(3,4)$. However, the destructive molecular events that follow TBI evolve over several days, and therefore there is a window of opportunity during which therapeutic strategies may improve outcome.

Autophagy is a highly regulated process that involves the degradation of a cell's cytoplasmic macromolecules and organelles. In mammalian cells, this catabolic mechanism utilizes the lysosomal system and has a homeostatic function in normal cell growth and development, helping to maintain a balance between the synthesis, degradation and subsequent recycling of cellular products $(5,6)$. Identification of autophagosomes remains the 'gold standard' method for the detection of autophagy (7). The first direct evidence that autophagy is increased following TBI, was reported in a study by Lai et al, which used a controlled cortical impact model of TBI to investigate this effect (8). More recently, a study administered a selective inhibitor of autophagy, 3-methyladenine, in a rat model of transient focal cerebral ischemia. They identified that this agent reduced infarct size, as 
compared with the vehicle treatment, which had no effect (9). However, 3-methyladenine has other effects, including inhibition of non-class III PI3-kinases and promotion of glycogen breakdown in hepatocytes (10).

Chloroquine (CQ) has long been used in the treatment and prevention of malaria, and less commonly has been employed in the treatment of autoimmune diseases, due to its immunosuppressive properties $(11,12)$. Recently, CQ has been recognized as an inhibitor of autophagy, and thus has been used as a pharmacological tool to study the role of autophagy in the laboratory $(13,14)$. CQ has also been used in clinical trials to evaluate its efficacy as an adjuvant to cancer therapeutic regimens (15). Inhibition of lysosome activity by $\mathrm{CQ}$ arrests the late stages of autophagy, including the degradation of the autolysosome, which prevents the supply of energy to the cell through the autophagy pathway (16). Recent studies have revealed that $\mathrm{CQ}$ is emerging as a potential therapeutic target in acute and chronic neurological disorders, including brain ischemia and Alzheimer's disease $(17,18)$. Nevertheless, no studies have examined the potential for CQ to provide neuroprotection in an animal model of TBI.

This study was designed to investigate the hypothesis that CQ has neuroprotective effects, via the attenuation of autophagy and inflammation, in a rat model of TBI.

\section{Materials and methods}

Animals. A total of 150 Sprague-Dawley rats (obtained from Hebei United University Experimental Animal Center, Tangshan, Hebei, China), weighing 280-320 g, were housed under a $12 \mathrm{~h}$ light/dark cycle with regular food and water supply. All procedures were performed in accordance with the institutional guidelines for the care and use of laboratory animals (Hebei Medical University, Shijiazhuang, China), and conformed to the National Institutes of Health (NIH) Guide for the Care and Use of Laboratory Animals (NIH Publication no. 80-23, revised 1996).

Models of TBI. The rat model of TBI was induced using a modified weight-drop device, as described previously by Marmarou et al (19). Briefly, rats were anesthetized with sodium pentobarbital (Nembutal, $60 \mathrm{mg} / \mathrm{kg}$ ) prior to surgery. A midline incision was made to expose the skull between the bregma and lambda suture lines and a steel disc $(10 \mathrm{~mm}$ in diameter and $3 \mathrm{~mm}$ thickness) was adhered to the skull using dental acrylic. Following this, rats were placed on a foam mattress underneath a weight-drop device, where a $450 \mathrm{~g}$ weight falls freely though a vertical tube from $1.5 \mathrm{~m}$ onto the steel disk. The sham-operated animals underwent the same surgical procedure, however they did not undergo TBI. Following the surgery, the rats received supporting oxygenation with $95 \% \mathrm{O}_{2}$ for no longer than 2 min and were then returned to their cages. All of the rats were housed in individual cages and placed on heat pads $\left(37^{\circ} \mathrm{C}\right)$ for $24 \mathrm{~h}$, to maintain normal body temperature during the recovery period.

Group and drug administration. Rats were randomly assigned to the sham-operated group (sham, $n=30$ ), TBI treated with CQ group $(\mathrm{CQ}, \mathrm{n}=60)$ and $\mathrm{TBI}$ received only equal volumes of $0.9 \%$ saline solution (vehicle, $\mathrm{n}=60$ ). $\mathrm{CQ}$ was dissolved in $0.9 \%$ saline and stored at $4{ }^{\circ} \mathrm{C}$. Following brain injury in the CQ group, CQ was immediately administered as an intraperitoneal injection ( $3 \mathrm{mg} / \mathrm{kg}$ body weight). All investigations were blind and the animal codes were revealed only at the end of the behavioral and histological analyses.

Immunofluorescence. Brain tissues were fixed in $4 \%$ paraformaldehyde for $24 \mathrm{~h}$, removed into $30 \%$ sucrose solution with $0.1 \mathrm{~mol} / \mathrm{l}$ phosphate-buffered saline (PBS; $\mathrm{pH}$ 7.4) until sinking to the bottom. Sections (200 $\mu \mathrm{m}$ apart) from the anterior to posterior hippocampus (bregma, 1.9-3 $\mathrm{mm}$ ) were made from the TBI rats and then embedded in OCT. Frozen sections $(15 \mu \mathrm{m})$ were sliced with a frozen slicer, treated with $0.4 \%$ Triton X-100 for $10 \mathrm{~min}$ and blocked in normal donkey serum for $1 \mathrm{~h}$. For double labeling, the frozen sections were incubated with a mixture of rabbit anti-microtubule-associated protein 1 light chain 3 (LC3) polyclonal antibody (Santa Cruz Biotechnology, Inc., Santa Cruz, CA, USA; dilution, 1:100) and mouse anti-neuron-specific nuclear protein $(\mathrm{NeuN})$ polyclonal antibody (Santa Cruz Biotechnology, Inc.; dilution, 1:100) overnight at $4^{\circ} \mathrm{C}$. The next day, sections were incubated with a mixture of fluorescein-conjugated anti-rabbit IgG and anti-mouse IgG (Santa Cruz Biotechnology, Inc.; dilution, 1:1,000) for $2 \mathrm{~h}$ at $37^{\circ} \mathrm{C}$ in the dark. All cell nuclei were counterstained by 4',6-diamidino-2-phenylindole (DAPI). Photographs were taken in a laser scanning confocal microscope (Olympus FV1000; Olympus Corporation, Tokyo, Japan). Primary antibodies were replaced with PBS in the negative control group.

Western blot analysis. Briefly, rats were anesthetized and underwent intracardiac perfusion with $0.1 \mathrm{~mol} / 1 \mathrm{PBS}(\mathrm{pH} 7.4)$. The hippocampal region of each rat brain was rapidly isolated, total proteins were extracted and protein concentration was determined by the BCA reagent (Beijing Solarbio Science \& Technology Co., Ltd, Beijing, China) method. Samples were subjected to sodium dodecyl sulfate polyacrylamide gel electrophoresis (SDS-PAGE). Separated proteins on the gel were transferred onto PVDF membranes (Roche Diagnostics, Mannheim, Germany). Blots were blocked with 5\% fat-free dry milk for $1 \mathrm{~h}$ at room temperature. Following blocking, the membrane was incubated with the primary antibodies overnight at $4^{\circ} \mathrm{C}$, including rabbit anti-LC3 polyclonal antibodies (Santa Cruz Biotechnology, Inc.; dilution, 1:500), rabbit anti-interleukin (IL)-1 $\beta$ polyclonal antibody (Santa Cruz Biotechnology, Inc.; dilution, 1:500), rabbit anti-tumor necrosis factor (TNF)- $\alpha$ polyclonal antibody (Santa Cruz Biotechnology, Inc.; dilution, 1:500), mouse anti- $\beta$-actin monoclonal antibody (Santa Cruz Biotechnology, Inc.; dilution, 1:500). The antibodies were then incubated with horseradish peroxidase conjugated anti-rabbit IgG and anti-mouse IgG (Cell Signaling Technology, Inc., Danvers, MA, USA; dilution, 1:5,000) for $2 \mathrm{~h}$ at room temperature. Following incubation with a fully titrated second antibody, the immunoblot on the membrane was visible. After development with an enhanced chemiluminescence (ECL) detection system, the densitometric signals were quantified using an imaging program. Immunoreactive bands of the protein expression were normalized to the intensity of corresponding bands for $\beta$-actin. The western blot analysis results were analyzed with NIH Image 1.41 software (Bethesda, MD, USA). 
Evaluation of brain edema. Brain edema was evaluated by analysis of brain water content as previously described (20). Rat brains were separated and weighed immediately with a chemical balance to obtain the wet weight (WW). Following drying in a desiccating oven for $24 \mathrm{~h}$ at $100^{\circ} \mathrm{C}$, dry tissues were weighed again to obtain the constant dry weight (DW). The percentage of water in the tissues was calculated according to the formula: \% brain water $=[(\mathrm{WW}-\mathrm{DW}) / \mathrm{WW}] \times 100$.

Recovery of motor function. The neurobehavioral status of the rats was evaluated using a set of 10 tasks, collectively termed the Neurologic Severity Score (NSS), which tests reflexes, alertness, coordination and motor abilities. One point is awarded for failure to perform a particular task, thus, a score of ten reflects maximal impairment, whereas a normal rat scores zero (21). Post-injury, NSS was evaluated at day 1, 3, 7 and 14. Each animal was assessed by an observer who was blinded to the type of treatment the animal had received. The difference between the initial NSS and that at a later time was calculated for each rat, and this value ( $\triangle \mathrm{NSS}$ ) reflected the spontaneous or treatment-induced recovery of motor function.

Morris water maze test. The spatial learning ability was assessed in a Morris water maze as described previously (22). The Morris water maze consists of a black circular pool (180 cm diameter, $45 \mathrm{~cm}$ high) filled with water $(30 \mathrm{~cm}$ depth) at $26^{\circ} \mathrm{C}$ and virtually divided into four equivalent quadrants: north $(\mathrm{N})$, west $(\mathrm{W})$, south (S) and east (E). A $2 \mathrm{~cm}$ submerged escape platform (diameter $12 \mathrm{~cm}$, height $28 \mathrm{~cm}$, made opaque with paint) was placed in the middle of one of the quadrants equidistant from the sidewall and the center of the pool. Rats were trained to find the platform prior to TBI or sham operation. For each trial, the rat was randomly placed into a quadrant start point $(\mathrm{N}, \mathrm{S}, \mathrm{E}$ or $\mathrm{W})$ facing the wall of the pool and was allowed a maximum of $60 \mathrm{sec}$ to escape to the platform. Rats that failed to escape within $90 \mathrm{sec}$ were placed on the platform for a maximum of $20 \mathrm{sec}$ and returned to the cage for a new trial (intertrial interval, $20 \mathrm{sec}$ ). Maze performance was recorded using a video camera suspended above the maze and interfaced with a video tracking system (HVS Imaging, Hampton, UK). The average escape latency of a total of five trials was calculated. This test was conducted at 3,7 and 14 days following trauma or sham operation.

Statistical analysis. All data were presented as the mean \pm standard error (SE). SPSS 16.0 (SPSS Inc., Chicago, IL, USA) was used for statistical analysis of the data. Statistical analysis was performed using one-way analysis of variance and followed by the Student-Newman-Keuls post-hoc test. $\mathrm{P}<0.05$ was considered to indicate a statistically significant result.

\section{Results}

Neurological deficit following TBI. As previously described by Marmarou et al (19), animals showed no significant difference in baseline locomotor activity for any parameters, including horizontal activity, vertical activity, total distance or stereotypy prior to surgery. Following the induction of
A
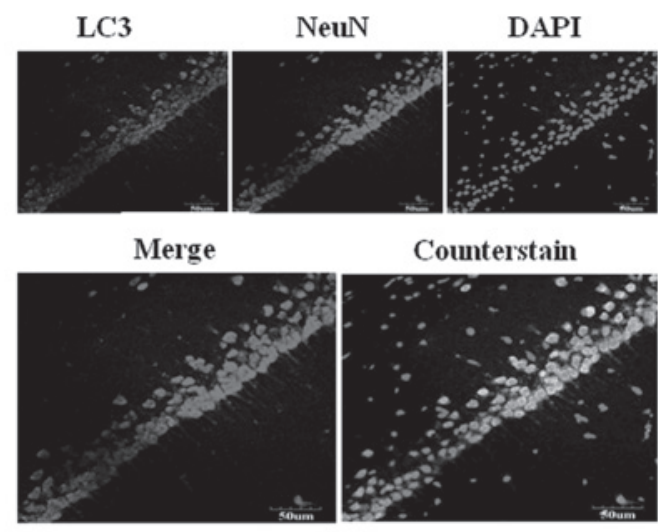

B

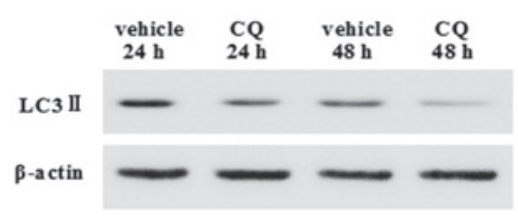

C

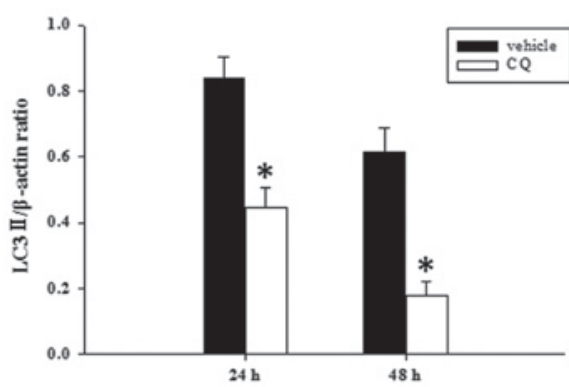

Figure 1. (A) Co-localization of NeuN and LC3 at $24 \mathrm{~h}$ following TBI was determined by immunofluorescent staining (magnification, $\mathrm{x} 400$ ) and cell nuclei were counterstained by DAPI. (B) Western blot analysis demonstrates levels of LC3-II and $\beta$-actin in the hippocampal sections at 24 and $48 \mathrm{~h}$ following TBI or CQ treatment. (C) Densitometry of the LC3-II band correlated to the $\beta$-actin band. The bars represent the mean $\pm \mathrm{SE}(\mathrm{n}=5)$. Results demonstrated that administration of CQ significantly decreased the level of LC3-II protein expression at 24 and $48 \mathrm{~h}$ following TBI ( ${ }^{*} \mathrm{P}<0.05 \mathrm{vs}$. TBI group) NeuN, neuron-specific nuclear protein; LC3, microtubule-associated protein 1 light chain 3; TBI, traumatic brain injury; DAPI, 4',6-diamidino-2-phenylindole; $\mathrm{CQ}$, chloroquine; SE, standard error.

injury, rats exhibited moderate to severe neurological deficits, including forelimb upon lifting the animal by its tail, decreased resistance to lateral push and reduced locomotor activity, flexion of contralateral torso and loss of righting reflex. Any mice not exhibiting behavioral deficits consistent with the surgery were excluded from further study.

Treatment with CQ attenuates neurons autophagy in the hippocampus following TBI. Since it has been recently demonstrated that the expression of the autophagy marker protein, LC3, was markedly elevated at $24 \mathrm{~h}$ following TBI (8), the co-localization of NeuN and LC3 was followed with immunofluorescent staining at $24 \mathrm{~h}$. As summarized in Fig. 1A, the majority of autophagy induced following TBI, occurred in neurons. Then, we examined whether CQ treatment inhibited the expression of LC3-II, as determined by western blot analysis (Fig. 1B). As demonstrated in Fig. 1C, at 24 and $48 \mathrm{~h}$ following TBI, administration of CQ 
A
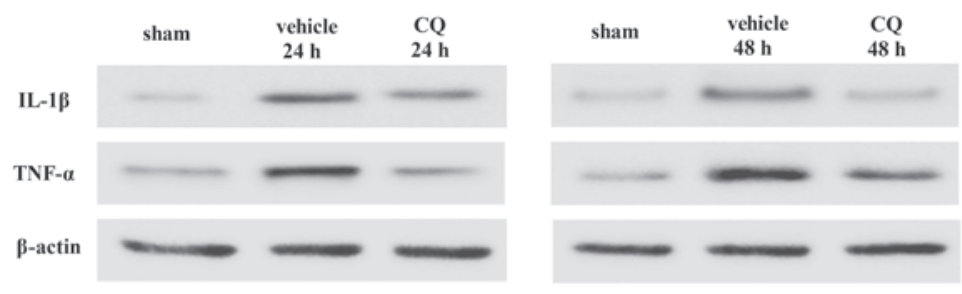

B
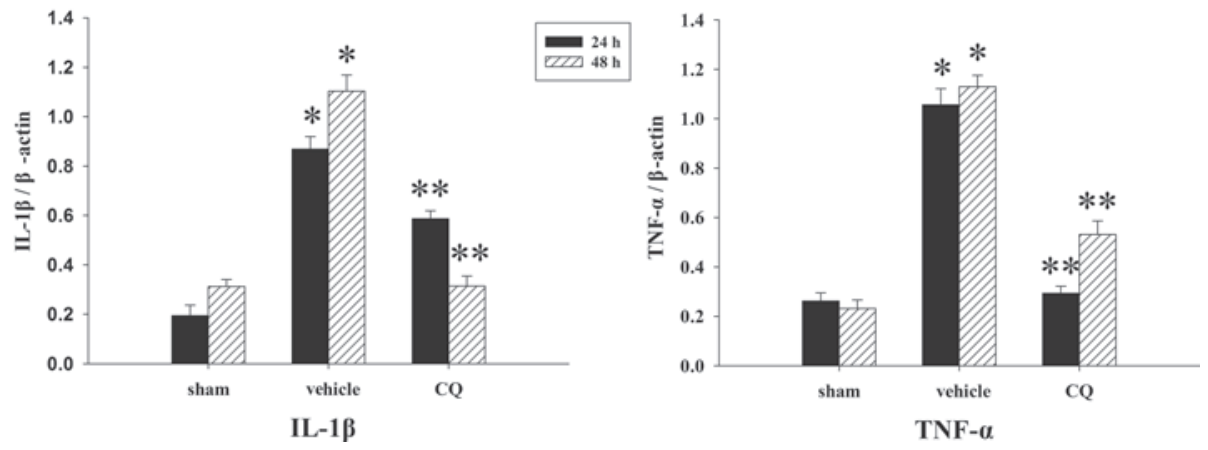

Figure 2. (A) Western blot analysis demonstrates levels of IL-1 $\beta$ and TNF- $\alpha$ in the hippocampus of rats at 24 and $48 \mathrm{~h}$ following TBI or sham operation. (B) The quantitative results of IL- $1 \beta$ and TNF- $\alpha$ were expressed as the ratio of densitometries of IL-1 $\beta$ and TNF- $\alpha$ to $\beta$-actin bands, expressed as the mean $\pm \mathrm{SE}$ ( $\mathrm{n}=5$ /group). The results demonstrated a significant increase of IL-1 $\beta$ and TNF- $\alpha$ expression in the TBI group ("P $<0.01$ vs. sham group). Treatment with CQ caused significant down-regulation of IL- $1 \beta$ and TNF- $\alpha$ expression at 24 and $48 \mathrm{~h}\left({ }^{* *} \mathrm{P}<0.05\right.$ vs. TBI group). IL-1, interleukin-1; TNF- $\alpha$, tumor necrosis factor- $\alpha$; TBI, traumatic brain injury; SE, standard error; $\mathrm{CQ}$, chloroquine.

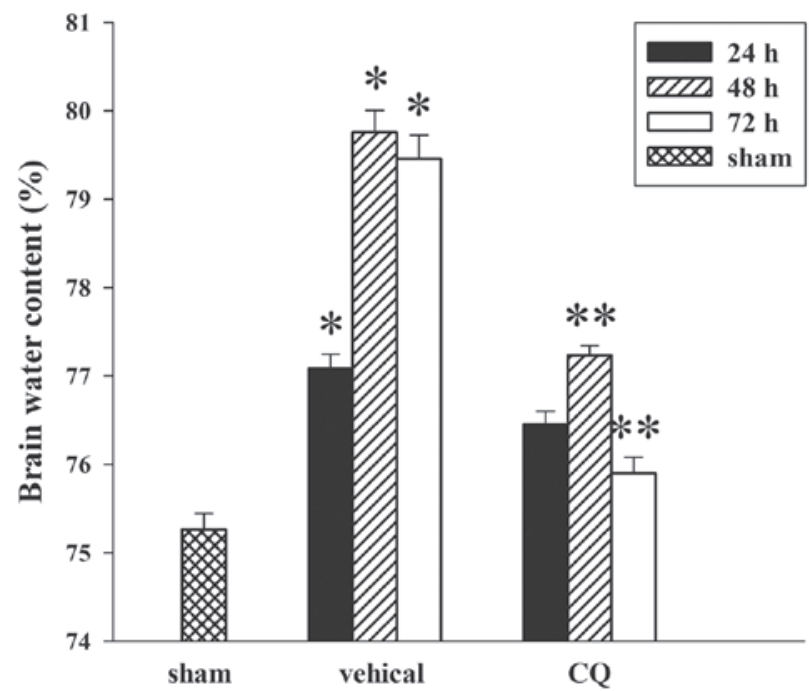

Figure 3. Effect of CQ on brain edema. Brain water content was determined at 24, 48 and $72 \mathrm{~h}$ following TBI and calculated as a percentage of DW and $\mathrm{WW}$ method. Bars represent the mean $\pm \mathrm{SE}$ ( $\mathrm{n}=5$ /group). Brain water content increased markedly at 24,48 and $72 \mathrm{~h}$ following TBI (" $\mathrm{P}<0.01 \mathrm{vs}$. sham group). Administration of CQ significantly decreased brain edema $\left({ }^{* *} \mathrm{P}<0.05\right.$ vs. TBI group) at 48 and $72 \mathrm{~h}$ as reflected by a decrease in brain water content. $\mathrm{CQ}$, chloroquine; TBI, traumatic brain injury; DW, dry weight; WW, wet weight; SE, standard error.

significantly attenuated the LC3-II protein expression in the rat hippocampus compared with the TBI group.

Treatment with $C Q$ attenuates inflammatory cytokine levels in the hippocampus following TBI. The expression levels of IL- $1 \beta$ and TNF- $\alpha$ in the hippocampus at 24 and $48 \mathrm{~h}$, were measured by western blot analysis (Fig. 2A). As revealed in

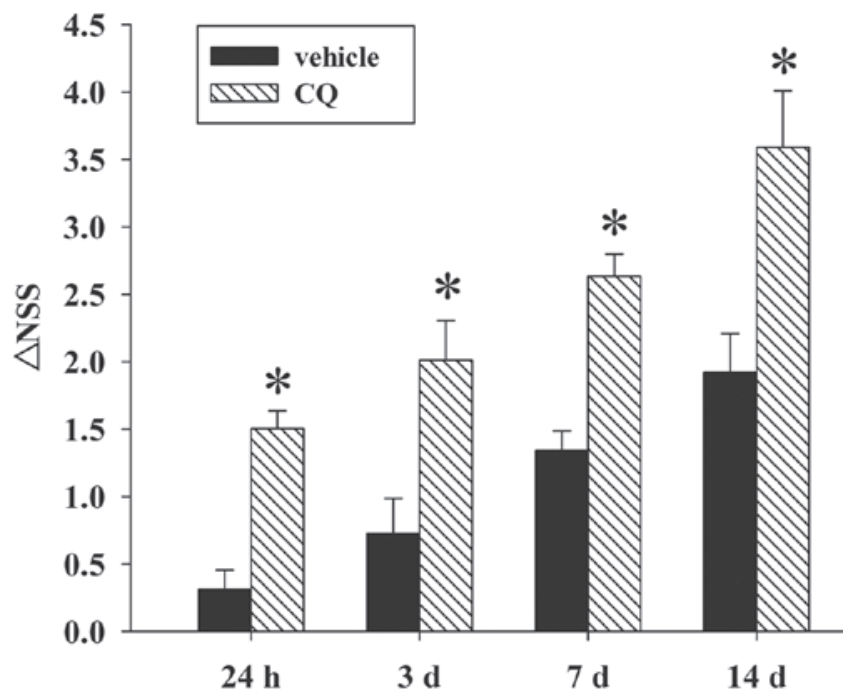

Figure 4. Effect of CQ on TBI-induced motor deficits. The temporal changes in motor recovery of the rat was determined at $24 \mathrm{~h}, 3,7$ and $14 \mathrm{~d}$ following TBI and calculated as $\triangle$ NSS. Bars represent the mean \pm SE ( $n=5 /$ group). Administration of CQ significantly improved motor function at $24 \mathrm{~h}, 3,7$ and $14 \mathrm{~d}$ following TBI $\left({ }^{* *} \mathrm{P}<0.05\right.$ vs. TBI group), as reflected by an increase in $\triangle \mathrm{NSS}$. TBI, traumatic brain injury; $\mathrm{CQ}$, chloroquine; SE, standard error; NSS, Neurologic Severity Score; d, days.

Fig. 2B, the inflammatory cytokine levels in the sham rat hippocampus at each time point, following the induction of injury, were consistently presented in a low background. All measured cytokine levels exhibited significant increases from different time points in the TBI group. Our results revealed that administration of CQ produced significant reductions in the injury-induced upregulation of IL-1 $\beta$ and TNF- $\alpha$ expression. 


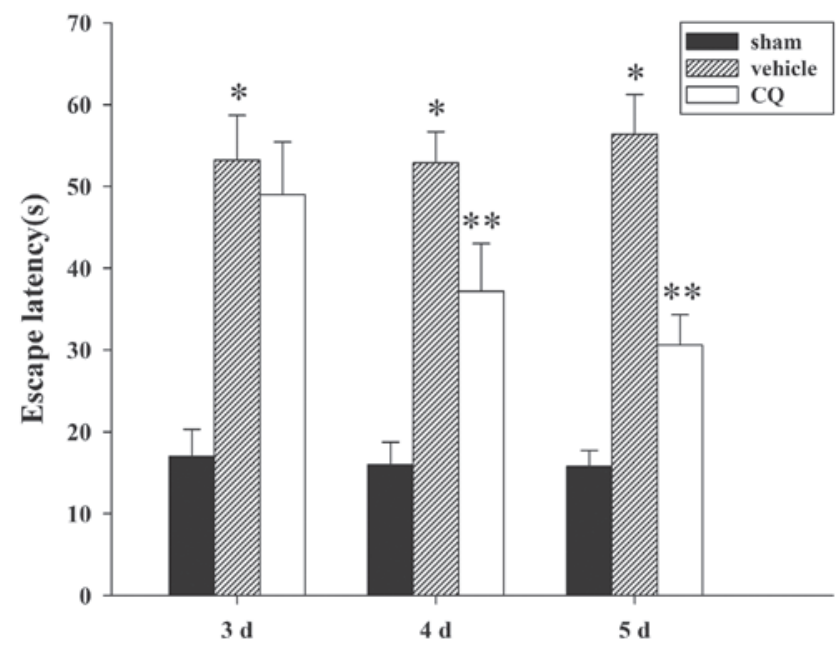

Figure 5. Effect of CQ on the escape latency performance via Morris water maze at 3, 4 and $5 \mathrm{~d}$ following TBI or sham operation. Bars represent the mean \pm standard error ( $n=5 /$ group). The escape latency increased markedly at 3,4 and $5 \mathrm{~d}$ following TBI ( ${ }^{*} \mathrm{P}<0.01$ vs. sham group). Treatment with CQ significantly reduced the time to identify the platform at 4 and $5 \mathrm{~d}\left({ }^{* *} \mathrm{P}<0.05\right.$ vs. TBI group). TBI, traumatic brain injury; CQ, chloroquine; $d$, days .

Treatment of CQ attenuates TBI-induced cerebral edema. The wet-dry weight method was used to evaluate brain edema. As shown in Fig. 3, CQ post-injury administration attenuated cerebral edema following TBI. In the TBI group, brain water content was significantly increased compared with the sham group at 24, 48 and $72 \mathrm{~h}$ after trauma. Furthermore, tissue water content in the CQ treatment group was significantly reduced at 48 and $72 \mathrm{~h}$ compared with the TBI group at the same time points.

Treatment of $C Q$ attenuates TBI-induced motor deficits. Fig. 4 depicts the temporal changes in functional recovery of the rat, expressed as $\triangle \mathrm{NSS}$. It is clear that post-injury administration of CQ significantly improved the motor function recovery of the trauma rats between $24 \mathrm{~h}$ and 14 days following TBI.

Treatment of $C Q$ attenuates TBI-induced learning and spatial memory function. Since CQ treatment was able to attenuate brain edema and improve motor deficits, we next examined whether CQ treatment could improve spatial learning function, as assessed by the Morris water maze at day 3, 7 and 14 following TBI or sham operation. As demonstrated in Fig. 5, TBI caused a significant spatial learning deficit at 3,7 and 14 days compared with the sham group, and CQ treatment significantly reduced the escape latency at 7 and 14 days compared with the TBI group.

\section{Discussion}

TBI is caused by immediate brain tissue disruption (primary injury) and post-injury cellular and molecular events (secondary injury). Primary damage is due to mechanical factors and occurs immediately at the moment of injury. Secondary injury is delayed and is produced by complex processes that are initiated at the moment of impact, but does not present clinically for a period of hours to days following the injury. This delayed pathophysiological cascade is now believed to result from a combination of factors, including increases in neurotransmitter release (23), free radical overproduction (24), exacerbated inflammatory response (25) and subsequent neuronal cell death via apoptosis and autophagy $(8,26)$.

In the present study, we investigated the efficacy of the autophagy inhibitor, CQ, as a therapeutic strategy for the acute treatment of TBI. It was identified that a single injection of CQ immediately following TBI, is neuroprotective in rats. The antimalarial drug CQ has been recognized as an autophagy inhibitor in a variety of disorders, including Alzheimer's disease and brain ischemia (15-18). Using a rat model of TBI, we have confirmed and extended these earlier observations, demonstrating for the first time, to the best of our knowledge, that post-injury administration of CQ improves behavioral outcomes and attenuates secondary cerebral edema following experimentally induced TBI in rats.

The use of animal models of TBI is essential for investigating and understanding the complex physiological and behavioural changes following injury. There are numerous established animal models of TBI, including rigid indentation, fluid percussion, impact acceleration, weight-drop and dynamic cortical deformation (27). However, head injury is a spontaneous, unpredictable event and no single animal model is entirely successful in reproducing the complete spectrum of pathological changes observed following TBI in humans (27). In this study, we used Marmarou's weight-drop model, which is currently the most commonly used to produce direct focal cortical compression in vivo, for its advantages of being simple and easy to operate. Nevertheless, there are limitations associated with this model, including variation of impact velocity by the falling $450 \mathrm{~g}$ steel column and the possibility of a rebound impact which together may result in the biomechanical data failing to accurately describe the resulting brain deformations. Therefore, any mice not exhibiting moderate to severe neurological deficits consistent with the surgery, were excluded from further study.

The first study to demonstrated that TBI may stimulate autophagy was conducted by Diskin et al in 2005 (28). This laboratory later evaluated the effects of treatment with the autophagy-inducer, rapamycin, in the closed head injury model. They identified that following TBI, intraperitoneal injection of rapamycin resulted in improved neurobehavioral function as determined by an NSS and increased neuronal survival in the injured region (29). Collectively, these data support the hypothesis that rapamycin-enhanced autophagy produces beneficial neurological effects following TBI. By contrast, Lai et al have demonstrated that systemic administration of the antioxidant, $\gamma$-glutamylcysteine ethyl ester (GCEE), following TBI, resulted in reduced autophagy as determined by LC3-II formation, which improved cognitive performance and reduced histological damage (8). Therefore, the role of autophagy after TBI, whether beneficial or detrimental, remains controversial.

In this study, we demonstrated that post-injury administration of the autophagy inhibitor, CQ, improved behavioral outcomes and attenuated secondary cerebral edema following TBI in rats. Our results are consistent with the findings of Lai et al, and it is therefore conceivable to hypothesize that the mechanism underlying the neuroprotective effects of CQ on TBI, is through the prevention of autophagic neuronal death. Furthermore, it appears these protective effects may also be 
explained by CQ's anti-inflammatory properties. It has been demonstrated that CQ inhibits TNF- $\alpha$, IL-1 and IL-6 production from mononuclear phagocytes $(30,31)$. In the present study, we observed that the expression of the inflammatory cytokines, IL-1 $\beta$ and TNF- $\alpha$, in the injured rat hippocampus of brain was suppressed by $\mathrm{CQ}$, as determined by western blot analysis. Hence, we suggest that the suppression of inflammatory cytokines following TBI may be associated with the neuroprotective effects of CQ treatment.

It has been hypothesized that CQ, rapamycin and GCEE, are not specific modulators of autophagy activity, but that these agents have a variety of other effects on cellular functions (31-33). Thus, targeting the specific processes of autophagy requires further investigation.

In summary, this study demonstrated that neuronal autophagy was inhibited by post-injury treatment of CQ in a rat model of TBI. Furthermore, CQ attenuates secondary brain edema and improves cognitive functioning. These findings emphasize that CQ administered immediately following injury, could be neuroprotective against the damaging consequences of TBI, and we anticipate that this study has shed light on the potential use of $\mathrm{CQ}$ as a neuroprotective agent in the treatment of cerebral injuries.

\section{Acknowledgements}

This study was supported by the grant from the Natural Science Foundation of Hebei, China (grant nos. H2012401071 and $\mathrm{H} 2014105079)$.

\section{References}

1. Thurman DJ, Alverson C, Dunn KA, Guerrero J and Sniezek JE: Traumatic brain injury in the United States: a public health perspective. J Head Trauma Rehabil 14: 602-615, 1999.

2. Davis AE: Mechanisms of traumatic brain injury: biomechanical, structural and cellular considerations. Crit Care Nurs Q 23: 1-13, 2000.

3. Wang YQ, Wang L, Zhang MY, et al: Necrostatin-1 suppresses autophagy and apoptosis in mice traumatic brain injury model. Neurochem Res 37: 1849-1858, 2012.

4. Luo CL, Chen XP, Yang R, et al: Cathepsin B contributes to traumatic brain injury-induced cell death through a mitochondria-mediated apoptotic pathway. J Neurosci Res 88: 2847-2858, 2010.

5. Shintani T and Klionsky DJ: Autophagy in health and disease: a double-edged sword. Science 306: 990-995, 2004.

6. Mizushima N: Autophagy: process and function. Genes Dev 21: 2861-2873, 2007.

7. Galluzzi L, Aaronson SA, Abrams J, et al: Guidelines for the use and interpretation of assays for monitoring cell death in higher eukaryotes. Cell Death Differ 16: 1093-1107, 2009.

8. Lai Y, Hickey RW, Yaming Chen Y, et al: Autophagy is increased after traumatic brain injury in mice and is partially inhibited by the antioxidant gamma-glutamylcysteinyl ethyl ester. J Cereb Blood Flow Metab 28: 540-550, 2008.

9. Puyal J, Vaslin A, Mottier V and Clarke PG: Postischemic treatment of neonatal cerebral ischemia should target autophagy. Ann Neurol 66: 378-389, 2009.

10. Caro LH, Plomp PJ, Wolvetang EJ, Kerkhof C and Meijer AJ 3-Methyladenine, an inhibitor of autophagy, has multiple effects on metabolism. Eur J Biochem 175: 325-329, 1988.
11. Homewood CA, Warhurst DC, Peters W and Baggaley VC: Lysosomes, $\mathrm{pH}$ and the anti-malarial action of chloroquine. Nature 235: 50-52, 1972.

12. Slater AF: Chloroquine: mechanism of drug action and resistance in Plasmodium falciparum. Pharmacol Ther 57: 203-235, 1993.

13. Janku F, McConkey DJ, Hong DS and Kurzrock R: Autophagy as a target for anticancer therapy. Nat Rev Clin Oncol 8: 528-539, 2011.

14. Lopez G, Torres K and Lev D: Autophagy blockade enhances HDAC inhibitors' pro-apoptotic effects: potential implications for the treatment of a therapeutic-resistant malignancy. Autophagy 7: 440-441, 2011.

15. Sotelo J, Briceño E and López-González MA: Adding chloroquine to conventional treatment for glioblastoma multiforme: a randomized, double-blind, placebo-controlled trial. Ann Intern Med 144: 337-343, 2006.

16. Kimura T, Takabatake Y, Takahashi A and Isaka Y: Chloroquine in cancer therapy: a double-edged sword of autophagy. Cancer Res 73: 3-7, 2013.

17. Liu C, Gao Y, Barrett J and Hu B: Autophagy and protein aggregation after brain ischemia. J Neurochem 115: 68-78, 2010.

18. Zhang JY, Peng C, Shi H, Wang S, Wang Q and Wang JZ: Inhibition of autophagy causes tau proteolysis by activating calpain in rat brain. J Alzheimers Dis 16: 39-47, 2009.

19. Marmarou A, Foda MA, van den Brink W, Campbell J, Kita $\mathrm{H}$ and Demetriadou K: A new model of diffuse brain injury in rats. J Neurosurg 80: 291-300, 1994.

20. Tang J, Liu J, Zhou C, et al: Mmp-9 deficiency enhances collagenase-induced intracerebral hemorrhage and brain injury in mutant mice. J Cereb Blood Flow Metab 24: 1133-1145, 2004

21. Beni-Adani L, Gozes I, Cohen Y, et al: A peptide derived from activity-dependent neuroprotective protein (ADNP) ameliorates injury response in closed head injury in mice. J Pharmacol Exp Ther 296: 57-63, 2001.

22. Hui-guo L, Kui L, Yan-ning Z and Yong-jian X: Apocynin attenuate spatial learning deficits and oxidative responses to intermittent hypoxia. Sleep Med 11: 205-212, 2010.

23. Yi JH and Hazell AS: Excitotoxic mechanisms and the role of astrocytic glutamate transporters in traumatic brain injury. Neurochem Int 48: 394-403, 2006.

24. Tyurin VA, Tyurina YY, Borisenko GG, et al: Oxidative stress following traumatic brain injury in rats. J Neurochem 75: 2178-2189, 2000.

25. Frugier T, Morganti-Kossmann MC, O'Reilly D and McLean CA: In situ detection of inflammatory mediators in post mortem human brain tissue after traumatic injury. J Neurotrauma 27: 497-507, 2010.

26. Raghupathi R: Cell death mechanisms following traumatic brain injury. Brain Pathol 14: 215-222, 2004.

27. O'Connor WT, Smyth A and Gilchrist MD: Animal models of traumatic brain injury: a critical evaluation. Pharmacol Ther 130: 106-113, 2011.

28. Diskin T, Tal-Or P, Erlich S, et al: Closed head injury induces upregulation of Beclin 1 at the cortical site of injury. J Neurotrauma 22: 750-762, 2005.

29. Erlich S, Alexandrovich A, Shohami E and Pinkas-Kramarski R: Rapamycin is a neuroprotective treatment for traumatic brain injury. Neurobiol Dis 26: 86-93, 2007.

30. van den Borne BE, Dijkmans BA, de Rooij H, le Cessie S and Verweij CL: Chloroquine and hydroxychloroquine equally affect tumor necrosis factor-alpha, interleukin 6 , and interferon-gamma production by peripheral blood mononuclear cells. J Rheumatol 24: 55-60, 1997.

31. Jang CH, Choi JH, Byun MS and Jue DM: Chloroquine inhibits production of TNF-alpha, IL-1beta and IL-6 from lipopolysaccharide-stimulated human monocytes/macrophages by different modes. Rheumatology (Oxford) 45: 703-710, 2006.

32. Fullerton HJ, Ditelberg JS, Chen SF, et al: Copper/zinc superoxide dismutase transgenic brain accumulates hydrogen peroxide after perinatal hypoxia ischemia. Ann Neurol 44: 357-364, 1998.

33. Sehgal S, Baker H and Vézina C: Rapamycin (AY-22,989), a new antifungal antibiotic. II. Fermentation, isolation and characterization. J Antibiot (Tokyo) 28: 727-732, 1975. 